PROSTATE CANCER

Hypoxiarelated gene panel is prognostic

A panel of 28 hypoxia-related genes has prognostic value for patients with localized prostate cancer. Translating this panel to the clinic has implications for treatment strategies.

Investigators first generated a prostatecancer-specific hypoxia signature by identifying changes in the regulation of hypoxia-related genes occurring both in vitro and in vivo. The final signature of 28 genes was derived by cross validating the prognostic significance of identified genes. Patients with a high signature score had significantly worse outcomes.

In the validation cohorts, increased signature score was associated with reduced biochemical-recurrence-free (BRF) survival in patients who received radical prostatectomy. For patients who received postprostatectomy plus radiotherapy or radiotherapy alone, the 28-gene signature was also prognostic for BRF survival. The score was useful in predicting benefit from hypoxia modification of radiotherapy in bladder cancer.

In a pooled cohort of patients with prostate cancer for whom metastatic outcome was assessed, the signature was predictive of distant metastatic events. Furthermore, hypoxia score was positively correlated with tumour stage and Gleason score.

This 28-gene signature had better prognostic significance than the Toustrup signature (which was derived from head and neck cancer, and was the only tested published signature to reach borderline significance in two prostate cancer cohorts). Addition of the signature to a 31-loci genomic classifier signature improved the prognostic utility of both scores.

These data show that this signature can be used to identify patients with hypoxic tumours who have poor outcomes, potentially influencing therapy.

Louise Stone

ORIGINAL ARTICLE Yang, L. et al. Development and validation of a 28-gene hypoxia-related prognostic signature for localized prostate cancer. EBiomed. https://doi.org/ 10.1016/j.ebiom.2018.04.019 (2018)
PROSTATE CANCER

\section{Stress less: targeting the stress response in advanced disease}

New data have revealed that one of the branches of the unfolded protein response to cell stress caused by oncogenic lesions is selectively activated in advanced prostate cancer. Targeting this mechanism induces cytotoxicity in metastatic disease.

Proteotoxic stress and accumulation of misfolded proteins caused by increased protein synthesis and the flux in the endoplasmic reticulum activates the unfolded protein response, which comprises three signalling arms (ATF6, IRE1,

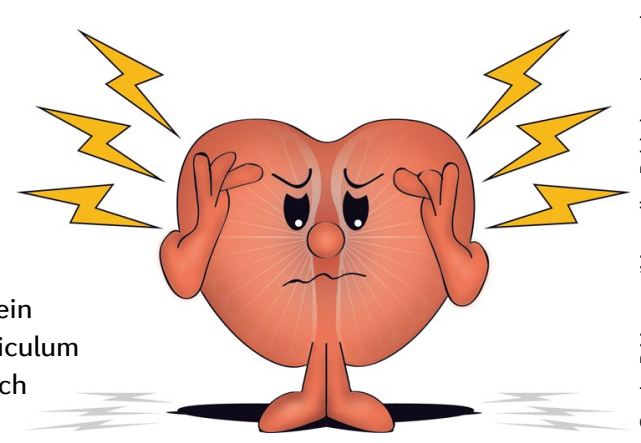
and PERK). However, the role of this response in cancer is poorly understood.

Modelling the distinct stages of prostate cancer in a transgenic mouse model showed that MYC overexpression alone did not cause adenocarcinoma, but did increase prostate cell proliferation. Mice with concomitant loss of PTEN developed adenocarcinoma within 10 weeks and had larger prostates than mice with PTEN loss alone. Global protein synthesis was increased by $\sim 20 \%$ in organoids derived from transgenic mouse prostates with either loss of PTEN or overexpression of MYC. However, global protein synthesis was significantly reduced in organoids with concomitant PTEN loss and MYC overexpression.

In tissue with PTEN loss plus MYC overexpression, immunofluorescent staining revealed 15-fold increased PERK phosphorylation compared with ATF6 and IRE1. Downstream signalling to P-eIF2 $\alpha$ and expression of ATF4 (a target of P-PERK-P-elF2 $\alpha$ signalling) were also increased. Treatment of organoids with a small-molecule inhibitor of P-eIF2 $\alpha$, integrated stress response inhibitor (ISRIB), increased synthesis of proteins in organoids with combined PTEN loss and MYC overexpression, whereas, in PTEN-deficient organoids, protein synthesis was not altered despite ISRIB inhibiting P-elF2 $\alpha$. Loss of PERK in mice with PTEN loss and MYC overexpression reduced prostate growth and cell proliferation and decreased cancer progression. P-elF2 $\alpha$ activation was also reduced in these mice.

A preclinical trial in which mice with established tumours received ISRIB or vehicle daily for 6 weeks showed that mice with PTEN loss and MYC overexpression had tumour regression after 3 weeks of treatment, whereas mice with PTEN deficiency alone had continued tumour growth.

In vitro, human cell lines that mimicked the molecular alterations in the mouse models (PTEN loss and MYC overexpression) showed increased PERK signalling and P-elF2 $\alpha$ expression. Treatment of these cells with ISRIB increased apoptosis.

Patient-derived xenograft (PDX) models from primary tumour and lymph node metastasis tissue were assessed for molecular alteration. The metastasis-derived PDXs had higher MYC and P-elF2 $\alpha$ expression than the PDXs from primary tissue, but both had PTEN loss. Treatment with ISRIB prolonged survival, caused tumour regression, and increased cell death and global protein synthesis in mice with metastasis-derived PDXs, whereas in mice with PDXs from primary tumour tissue, the effects of ISRIB were short lived and no change in global protein synthesis was observed. Increased survival was also seen in a mouse model of metastatic castration-resistant prostate cancer treated with ISRIB.

In patients with prostate cancer who received surgery with curative intent, those with tumours showing PTEN loss plus high MYC expression were more likely to experience metastatic progression than those with either PTEN loss or high MYC expression alone. In patients with PTEN loss and high P-elF2 $\alpha$ expression, metastasis-free and prostate-cancerspecific-mortality (PCSM)-free survival were reduced. However, high MYC expression with either low or high P-elF2 $\alpha$ expression was not associated with increased risk of PCSM or metastasis. High P-elF2 a expression and PTEN loss independently predicted risk of metastasis.

These data suggest that targeting the adaptive stress response (the activation of P-elF2a signalling that decreases global protein synthesis), which occurs in advanced prostate cancer, has therapeutic potential in this disease.

Louise Stone

ORIGINAL ARTICLE Nguyen, H. G. et al. Development of a stress response therapy targeting aggressive prostate cancer. Sci. Transl. Med. 10, eaar2036 (2018) 